\title{
Тензосопротивление $n$-Ge разной кристаллографической ориентации при наличии классически сильного магнитного поля и без него
}

\author{
(С) Г.П. Гайдар ${ }^{1}$, П.И. Баранский ${ }^{2}$ \\ ${ }^{1}$ Институт ядерных исследований Национальной академии наук Украины, \\ 03680 Киев, Украина \\ ${ }^{2}$ Институт физики полупроводников им. В.Е. Лашкарева Национальной академии наук Украины, \\ 03028 Киев, Украина \\ E-mail: gaydar@kinr.kiev.ua
}

(Получена 29 ноября 2016 г. Принята к печати 7 декабря 2016 г.)

Экспериментально и теоретически исследованы изменения тензосопротивления, тензомагнето- и магнетотензосопротивления в широких диапазонах напряженностей магнитного поля, $0 \leq H \leq 100$ кЭ, и механических напряжений, $0 \leq X \leq 0.7$ ГПа, при $77 \mathrm{~K}$ в условиях невырожденной статистики электронного газа в кристаллах $n$-Ge разной кристаллографической ориентации.

DOI: 10.21883/FTP.2017.07.44657.8465

\section{1. Введение}

Энергетическая эквивалентность долин многодолинного полупроводника типа $n$-Ge или $n$-Si может быть нарушена при упругом деформировании кристалла в направлении, по отношению к которому изоэнергетические эллипсоиды расположены несимметрично $[1,2]$. Актуальность этого обстоятельства впервые была обнаружена в опытах Смита [3] по изучению эффекта тензосопротивления в кристаллах германия и кремния. Херринг [4] впервые показал, что наблюдаемое изменение удельного сопротивления многодолинных полупроводников при одноосной упругой деформации связано с деформационным нарушением энергетической эквивалентности изоэнергетических эллипсоидов в таких опытах и последующим межминимумным (межэллипсоидным) перераспределением носителей заряда при неизменной их концентрации ( $n_{e}=$ const) в зоне проводимости. Смещение по шкале энергий эквивалентных (до наложения на кристалл деформирующих усилий) минимумов энергии зависит от направления оси деформации. Так, например, наложение на кристалл $n$-Ge сжимающего напряжения в направлении [111] приводит к тому, что изоэнергетический эллипсоид, ориентированный в этом направлении, понижается (опускается) на величину $-(1 / 3) E_{2} S_{44} X$ эВ (где $E_{2}=16.4$ эВ - деформационный потенциал, $S_{44}=1.47 \cdot 10^{-12} \mathrm{~cm}^{2} /$ дин - коэффициент податливости, $X-$ механическое напряжение в направлении оси деформации), тогда как остальные три эллипсоида поднимаются вверх по шкале энергий на величину $+(1 / 9) E_{2} S_{44} X$ эВ [5]. Межэллипсоидное перераспределение носителей заряда в соответствии с фактором Больцмана определяется относительным смещением эллипсоидов по шкале энергий (обусловливаемым направленной деформацией) и температурой [6].

Упругая направленная деформация приводит к изменению как межатомных расстояний, так и симметрии кристалла [7]. Однако значительные изменения в зонной структуре кристалла возникают лишь при выполнении условия $\Delta \varepsilon \gg k T$, где $\Delta \varepsilon$ соответствует межминимумному смещению, вызываемому направленной деформацией, $T$ - температура, $k-$ постоянная Больцмана.

При наложении на кристалл механического напряжения $X \neq 0$ вдоль определенного кристаллографического направления величину удельного сопротивления $\rho_{X}$, которое при этом измеряется в деформированном кристалле, называют тензосопротивлением; $\rho_{0}$ - удельное сопротивление недеформированного кристалла.

Если на исследуемые кристаллы одновременно влияет и одноосная упругая деформация $X$, и магнитное поле $H$, то величину

$$
\left.\frac{\Delta \rho_{H}^{\|}}{\rho_{0}}\right|_{X=\mathrm{const}} \equiv \frac{\rho_{X}^{H}-\rho_{X}^{0}}{\rho_{X}^{0}}=f(H),
$$

характеризующую изменение удельного сопротивления в зависимости от напряженности магнитного поля $H$ при постоянной величине деформирующего усилия $(X=$ const), называют магнетотензосопротивлением [5].

Величину

$$
\left.\frac{\Delta \rho_{X}^{\|}}{\rho_{0}}\right|^{H=\mathrm{const}} \equiv \frac{\rho_{X}^{H}-\rho_{0}^{H}}{\rho_{0}^{H}}=f(X),
$$

характеризующую изменение удельного сопротивления в зависимости от деформирующего усилия $X$ при постоянном магнитном поле $(H=\mathrm{const})$, называют тензомагнетосопротивлением [5].

Целью данной работы было исследование изменений тензосопротивления, тензомагнето- и магнетотензосопротивления в широких диапазонах напряженностей магнитного поля, $0 \leq H \leq 100$ кЭ, и механических напряжений, $0 \leq X \leq 0.7$ ППа, в кристаллах $n$-Ge различной кристаллографической ориентации.

\section{2. Результаты и обсуждение}

Зависимости удельного сопротивления и магнетотензосопротивления от одноосной упругой деформации в 


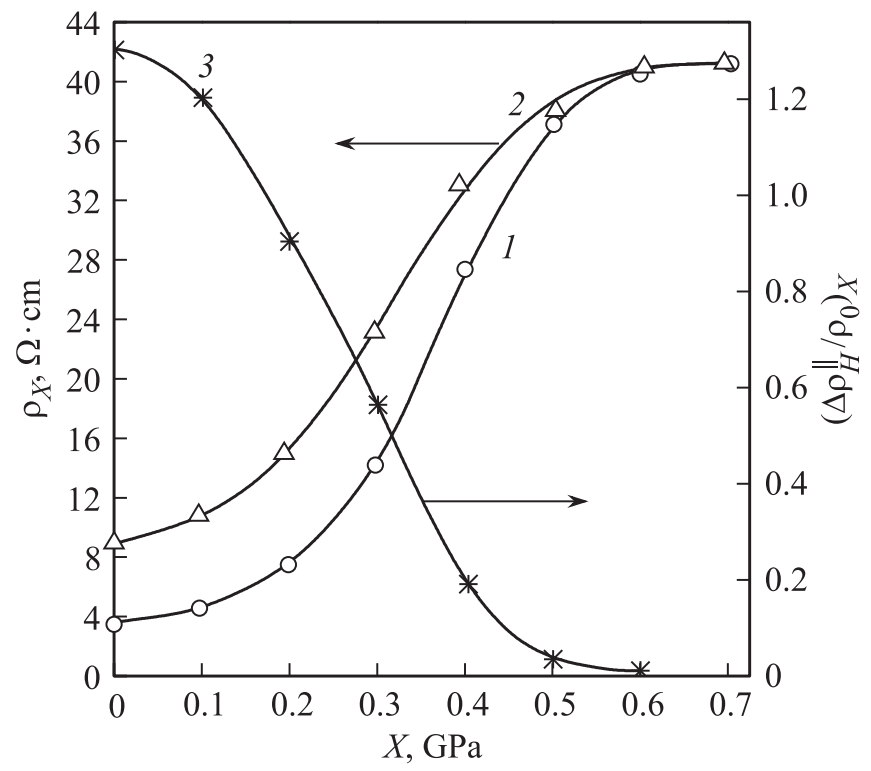

Рис. 1. Для образцов $n$-Ge $\left(n_{e}=6.5 \cdot 10^{13} \mathrm{~cm}^{-3}\right)$ зависимости от механического напряжения сжатия $X$ при $77 \mathrm{~K}: 1-$ удельного сопротивления $\rho_{X}^{0}$, измеренного вдоль оси сжатия [111] в отсутствие $H ; 2-$ значений $\rho_{X}^{H}$ в случае $\mathbf{X}\|\mathbf{H}\|[111]$ при $H=100$ кЭ; 3 - продольного магнетотензосопротивления $\left(\Delta \rho_{H}^{\|} / \rho_{0}\right)_{X} \equiv\left(\rho_{X}^{H}-\rho_{X}^{0}\right) / \rho_{X}^{0}$, измеренного при $\mathbf{X}\|\mathbf{J}\| \mathbf{H} \|[111]$ и $H=100$ кЭ $=$ const.

условиях $\mathbf{X}\|\mathbf{J}\| \mathbf{H} \|$ [111] ( $J$ - ток) были исследованы в $n$-Ge $\left(n_{e}=6.5 \cdot 10^{13} \mathrm{~cm}^{-3}\right)$ при температуре жидкого азота $(T=77 \mathrm{~K})$. Если механическое напряжение сжатия $X$, приложенное к кристаллу вдоль направления [111] $(\mathbf{X} \|[111])$, достаточно велико $(X=0.6$ ГПа), то электроны переходят в минимум, расположенный в направлении оси деформации и опускающийся под воздействием давления по шкале энергий. Поскольку все электроны переходят в минимум с наименьшей подвижностью $\mu=\mu_{\|}$, то удельное сопротивление кристалла $\rho(X)$ будет сильно возрастать с ростом сжимающих механических напряжений $X$ до насыщения (рис. 1 , кривая 1), определяемого выражением

$$
\rho_{X \rightarrow \infty}^{[111]} \equiv \rho_{X(\infty)}^{[111]}=\left(e n_{e} \mu_{\|}\right)^{-1}=\text { const, }
$$

где $e-$ заряд электрона, $n_{e} \equiv N-$ полная концентрация носителей заряда в кристалле, не зависящая от $X, \mu_{\|}-$подвижность электронов вдоль большой оси изоэнергетического эллипсоида, соответствующая максимальной эффективной массе $m_{\|}$.

Поскольку концентрация носителей заряда в исследуемых образцах $n$-Ge, полученная из холловских данных при учете холл-фактора, составляла $n_{e}=6.5 \cdot 10^{13} \mathrm{~cm}^{-3}$, величину подвижности вдоль большой оси изоэнергетического эллипсоида (вдоль оси давления) можно получить по значению $\rho_{X(\infty)}^{[111]}$, найденному из насыщения кривой 1 (рис. 1) и по формуле (3):

$$
\mu_{\|}=\frac{1}{e n_{e} \rho_{X(\infty)}^{[111]}}=2340 \mathrm{~cm}^{2} / \mathrm{B} \cdot \mathrm{c} \text {. }
$$

В [5] показано, что $\rho_{X(\infty)}^{[111]}$ и $\rho_{0}$ связаны с параметром анизотропии подвижности $K$ в отдельно взятом изоэнергетическом эллипсоиде простым соотношением:

$$
K=\frac{\mu_{\perp}}{\mu_{\|}}=\frac{K_{m}}{K_{\tau}}=\frac{3}{2} \frac{\rho_{X(\infty)}^{[111]}}{\rho_{0}}-\frac{1}{2},
$$

где $\rho_{0} \equiv \rho_{0}^{0}-$ удельное сопротивление образца при $X=0$ и $H=0, \mu_{\|}$и $\mu_{\perp}-$ подвижности носителей заряда вдоль и поперек большой оси изоэнергетического эллипсоида соответственно, $K_{m}=m_{\|} / m_{\perp}$ - параметр анизотропии эффективной массы, $m_{\|}$и $m_{\perp}-$ циклотронные эффективные массы для отдельно взятого изоэнергетического эллипсоида вдоль большой оси и перпендикулярно к ней соответственно, $K_{\tau}=\left\langle\tau_{\|}\right\rangle /\left\langle\tau_{\perp}\right\rangle-$ параметр анизотропии рассеяния, $\tau_{\|}$и $\tau_{\perp}-$ компоненты тензора времени релаксации в отсутствие магнитного поля в линейном приближении.

При деформировании кристаллов $n$-Ge направленным сжатием X $\|\mathbf{J}\|[111]$, используя экспериментально полученную величину $\rho_{X(\infty)}^{[111]} / \rho_{0}$ и соотношение $(5)$, можно найти значение параметра анизотропии подвижности $K=\mu_{\perp} / \mu_{\|}$при условии, что направленная деформация $n$-Ge обеспечивает лишь межминимумное перераспределение носителей заряда в зоне проводимости кристаллов, не изменяя при этом их полной концентрации. Таким образом, из выражения (5) с учетом данных рис. 1 была найдена величина параметра анизотропии подвижности $K=(15.95 \pm 0.04)$. Далее, зная $\mu_{\|}$и $K$, можно вычислить $\mu_{\perp}=\mu_{\|} K=37300 \mathrm{~cm}^{2} / \mathrm{B} \cdot$ с. Учитывая, что для $n$-Ge $K_{m}=m_{\|} / m_{\perp}=1.58 / 0.082 \approx 19.3$ [8], и используя полученное значение параметра $K=15.95$, находим параметр анизотропии рассеяния $K_{\tau}=K_{m} / K=1.21$.

Кривыми 1 и 2 (рис. 1) представлены зависимости от механического напряжения одноосного упругого сжатия $X$ удельного сопротивления кристалла $\rho_{X}^{0}=f(X)$ и удельного сопротивления кристалла в продольном магнитном поле $H=100$ кЭ $\rho_{X}^{H}=f(X)$ соответственно. Кривой 3 (рис. 1), изображающей зависимость $\Delta \rho_{H}^{\|} / \rho_{0}=f(X)$, представлено уменьшение магнетотензосопротивления (в условиях $\mathbf{X}\|\mathbf{J}\| \mathbf{H} \|[111]$ и $H=100$ кЭ = const) по мере перестройки зоны проводимости в однодолинную (при увеличении механического напряжения $X)$.

В соответствии с теорией гальваномагнитных эффектов в анизотропных полупроводниках [9], при расположении векторов $\mathbf{J}$ и $\mathbf{H}$ параллельно большой оси изоэнергетического эллипсоида изменение сопротивления одноэллипсоидного полупроводника в продольном (неквантующем) магнитном поле (подобно случаю сферически-симметричных изоэнергетических поверхностей) должно быть тождественно равным нулю. Поэтому при $\mathbf{X}\|\mathbf{J}\| \mathbf{H} \|[111]$ по мере приближения к условиям полного переселения носителей заряда (с ростом $X$ ) в эллипсоид, расположенный вдоль оси деформации [111], магнетотензосопротивле- 


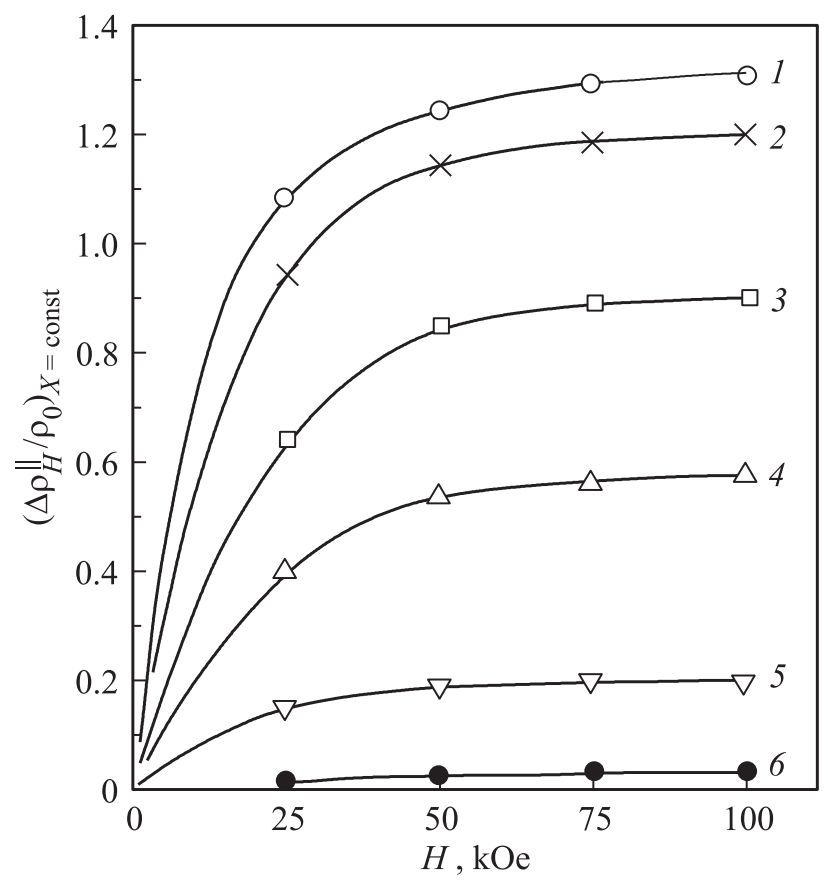

Рис. 2. Зависимости продольного магнетотензосопротивления от напряженности магнитного поля $H$ в $n$-Ge $\left(n_{e}=6.5 \cdot 10^{13} \mathrm{~cm}^{-3}\right)$ при $77 \mathrm{~K}$, измеренные в условиях $\mathbf{X}\|\mathbf{J}\| \mathbf{H} \|$ [111] при разных значениях $X$, ГПа: $1-0,2-0.1$, $3-0.2,4-0.3,5-0.4,6-0.5$; для $X=0.6$ ГПа и $H=100$ кЭ получено значение $\Delta \rho_{H}^{\|} / \rho_{0} \approx 0.015$.

ние $\Delta \rho_{H}^{\|} /\left.\rho_{0}\right|_{X=\text { const }} \equiv\left(\rho_{X}^{H}-\rho_{X}^{0}\right) / \rho_{X}^{0}$ должно уменьшаться и в пределе (т.е. при выходе тензосопротивления на насыщение) должно обращаться в нуль, что и дает эксперимент (рис. 1, кривая 3).

В правильности вывода о том, что магнетотензосопротивление в сильно деформированном кристалле практически равно нулю, можно убедиться и в другой постановке опытов. Например, можно измерять зависимость изменений $\rho$ от напряженности магнитного поля $H$ при разных значениях механической нагрузки $X$.

На рис. 2 представлены зависимости магнетотензосопротивления $\Delta \rho_{H}^{\|} /\left.\rho_{0}\right|_{X=\text { const }}=f(H)$ в классически сильных магнитных полях $H$ напряженностью до 100 кЭ $(\mu H / c \approx 30$ при $T=77 \mathrm{~K})$, измеренные при разных значениях $X=$ const в $n$-Ge. Из рис. 2 видно, что магнетосопротивление, измеренное при $X=0$ в магнитных полях $0 \leq H \leq 100$ кЭ (кривая 1 ), возрастает от 0 до максимальных значений. По мере переселения электронов в долину [111], опускающуюся в шкале энергий с ростом давления $X$, магнетотензосопротивление в продольном магнитном поле уменьшается (рис. 2, кривые 2-6).

Кривая 3 на рис. 1 представляет по существу сечение семейства зависимостей (рис. 2) для фиксированной величины $H=100$ КЭ = const. Из рис. 2, однако, лучше, чем из рис. 1 , видно, что даже при $X=0.6$ ГПа магнетотензосопротивление не точно равно нулю (в пределах точности эксперимента, составляющей для ме- тодик, которые используют импульсные магнитные поля, $\sim 5 \%$ ). При $H=100$ кЭ и $X=0.6$ ГПа получено значение $\Delta \rho_{H}^{\|} / \rho_{0} \approx 0.015$, что, по-видимому, связано с началом проявления эффектов квантования (поскольку $\hbar\left(e H / m_{e}^{*} c\right) \approx k T$ при $77 \mathrm{~K}$ и $H=100$ кЭ, где $\hbar-$ постоянная Планка, $c$ - скорость света, $e$ и $m_{e}^{*}-$ заряд и эффективная масса электрона соответственно).

Наряду с изучением для кристаллов $n$-Ge магнетотензосопротивления (при разных механических нагрузках $X=$ const, $\mathbf{X} \|[111])$ не менее важным является изучение тензомагнетосопротивления при $H=0,12.5,50$, 100 кЭ и $T=77 \mathrm{~K}$ (рис. 3, кривые $1-4$ ). Полученные результаты, на первый взгляд, как будто находятся в противоречии с данными (рис. 1, кривые 1 и 2), которые указывают на то, что при очень больших сжимающих напряжениях $X$ магнитное поле $\mathbf{H}$, ориентированное вдоль $\mathbf{X} \|[111]$, не изменяет удельное сопротивление кристалла $\left(\rho_{X(\infty)}^{H} \equiv \rho_{X(\infty)}^{0}\right)$. В то же время кривые (рис. 3) для тензомагнетосопротивления, измеряемого при различных значениях $H=$ const, наиболее сильно различаются в области максимальных значений $X$. Никакого противоречия, однако, здесь нет, если учесть, что на рис. 3 приведены в зависимости от $X$ значения не $\rho_{X}^{H}=f(X)$, а отношения $\rho_{X}^{H} / \rho_{0}^{H}$, числитель в котором действительно становится индифферентным по отношению к $H$ по мере возрастания $X$, а знаменатель, не зависящий от $X$, с ростом $H$ возрастает. Учет этого обстоятельства объясняет не только последовательность в расположении кривых $1-4$ на рис. 3 , но в равной мере и вид зависимости, представленной на рис. 1 .

Для сравнения проведены аналогичные исследования тензо- и магнетотензосопротивления кристаллов $n$-Ge, легированных примесью сурьмы $(\mathrm{Sb})$ $\left(n_{e}=1.48 \cdot 10^{13} \mathrm{~cm}^{-3}\right.$ и $\rho_{0}=9.96$ Ом $\cdot$ см при $\left.77 \mathrm{~K}\right)$, но

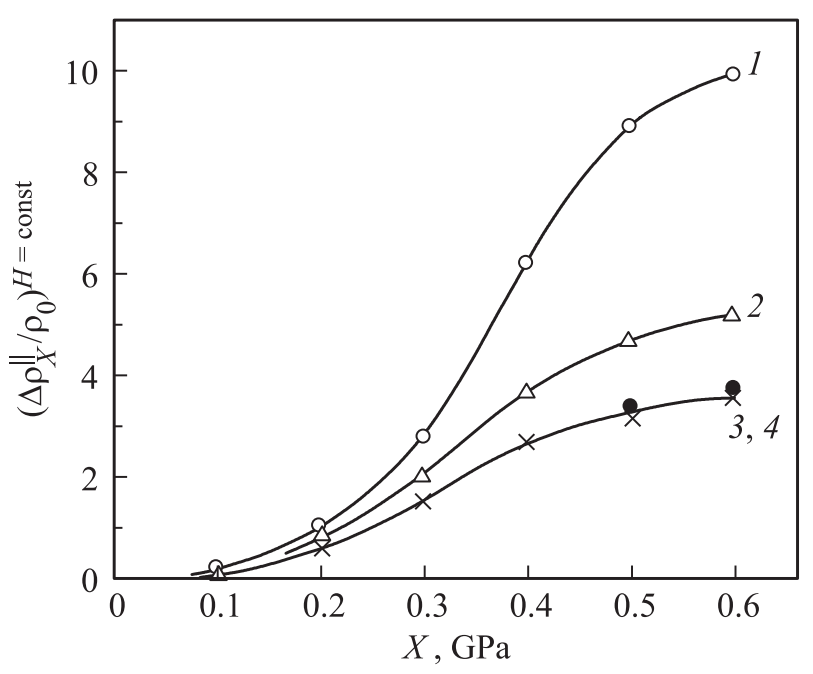

Рис. 3. Зависимости тензомагнетосопротивления $\left(\Delta \rho_{X}^{\|} / \rho_{0}\right)^{H=\mathrm{const}} \equiv\left(\rho_{X}^{H}-\rho_{0}^{H}\right) / \rho_{0}^{H}=f(X) \quad$ в $\quad n$-Ge $\quad\left(n_{e}=\right.$ $\left.=6.5 \cdot 10^{13} \mathrm{~cm}^{-3}\right)$ при $77 \mathrm{~K}$, измеренные в условиях $\mathbf{X}\|\mathbf{J}\| \mathbf{H} \|[111]$ при разных значениях $H$, кЭ: $1-0$, $2-12.5,3-50,4-100$. 


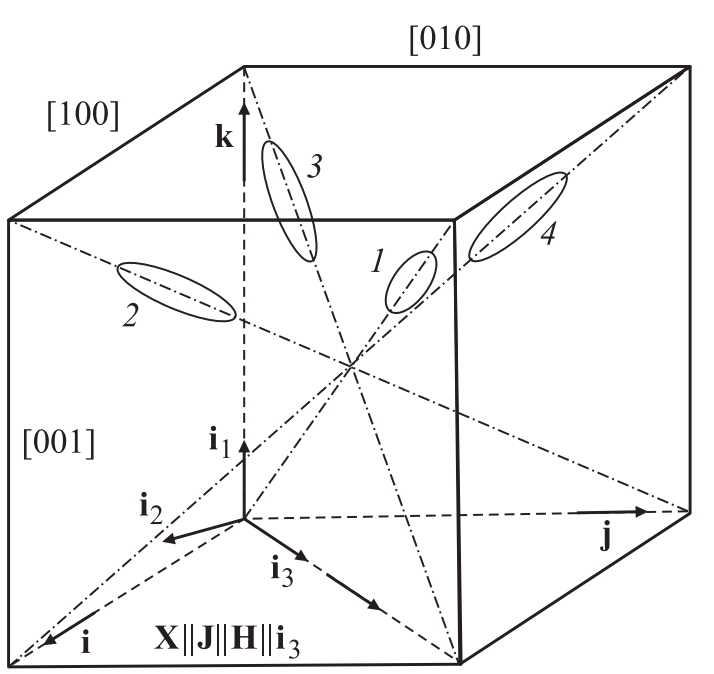

Рис. 4. Расположение лабораторной системы координат $\left(\mathbf{i}_{1}, \mathbf{i}_{2}, \mathbf{i}_{3}\right)$ по отношению к осям кристалла при изучении магнетотензосопротивления $n$-Ge в условиях $\mathbf{X}\|\mathbf{J}\| \mathbf{H} \|[110]$.

ориентированных в кристаллографическом направлении [110].

Рассмотрим распределение электронов по разным долинам, соответствующее этим условиям. Как следует из рис. 4, все долины по характеру их расположения относительно оси деформации можно разделить на две группы, а именно: долины 1 и 3 составляют первую группу, а долины 2 и 4 - вторую группу долин, эквивалентно расположенных относительно оси деформации и характеризуемых поэтому эквивалентными сдвигами по шкале энергий при наложении в направлении оси деформации механической нагрузки.

При $T=77 \mathrm{~K}$ и деформирующем усилии $X \approx 0.6-0.7$ ГПа в условиях сжатия кристалла почти все электроны будут сосредоточены в двух опустившихся по шкале энергий долинах 1 и 3 . В связи с этим основные особенности магнетотензосопротивления при $\mathbf{X}\|\mathbf{J}\| \mathbf{H} \|[110]$ должны качественно отличаться от ранее рассмотренного случая $\mathbf{X}\|\mathbf{J}\| \mathbf{H} \|[111]$, когда все носители заряда (при достаточно сильном сжатии) сосредоточивались в единственном изоэнергетическом эллипсоиде, ориентированном вдоль оси деформации. Так, например, вместо ранее наблюдавшегося (при $\mathbf{X}\|\mathbf{J}\| \mathbf{H} \|[111]$ ) уменьшения изменения сопротивления с возрастанием магнитного поля $H$ при увеличении деформации, в условиях $\mathbf{X}\|\mathbf{J}\| \mathbf{H} \|[110]$ можно ожидать сильного возрастания магнетотензосопротивления с ростом $X$, поскольку электроны в этом случае будут переходить из эллипсоидов 2 и 4 в эллипсоиды 1 и 3 , а магнитное поле Н направлено под углом к осям вращения последних.

В рассматриваемых условиях $n_{e}=4 N_{0}=2 N_{1}+2 N_{2}$, где $N_{1}$ - число электронов в долинах 1 и $3, N_{2}$ - число электронов в долинах 2 и 4 , а $N_{0}$ - число электронов в каждой из долин в недеформированном кристалле.
На образцах $n$-Ge $\langle\mathrm{Sb}\rangle$ при $\mathbf{X}\|\mathbf{J}\| \mathbf{H} \|[110]$ и температуре $T=77 \mathrm{~K}$ измерялись следующие величины:

- тензосопротивление $\rho_{X} \equiv \rho_{X}^{0}=f(X)$ в области $0 \leq X \leq 0.6$ ГПа при сжатии кристалла;

- магнетотензосопротивление $\left(\Delta \rho_{H}^{\|} / \rho_{0}\right)_{X=\mathrm{const}} \equiv$ $\equiv\left(\rho_{X}^{H}-\rho_{X}^{0}\right) / \rho_{X}^{0}=f(H) \quad$ в классически сильных магнитных полях $(12.5 \leq H \leq 100$ кЭ) при разных фиксированных значениях механического напряжения $X=$ const;

- тензомагнетосопротивление $\left(\Delta \rho_{X}^{\|} / \rho_{0}\right)^{H=\text { const }} \equiv$ $\equiv\left(\rho_{X}^{H}-\rho_{0}^{H}\right) / \rho_{0}^{H}=f(X)$ в широком диапазоне изменения $X \quad(0 \leq X \leq 0.6$ ГПа) при использовании фиксированных значений $H=0$ и 100 кЭ, которые служили при этом параметрами.

Результаты проведенных измерений изображены точками на рис. 5-7 соответственно. Сплошными линиями на этих рисунках показаны результаты расчетов зависимостей $\rho_{X}=f(X),\left(\Delta \rho_{H}^{\|} / \rho_{0}\right)_{X=\text { const }}=f(H)$, $\left(\Delta \rho_{X}^{\|} / \rho_{0}\right)^{H=\text { const }}=f(X)$, которые выполнялись по формулам теории анизотропного рассеяния, представленным в Приложении, с использованием констант деформационных потенциалов $\Xi_{u}=16.4$ эВ, $\Xi_{d}=-6.4$ эВ, полученных в работе [10]. Предполагалось, что одноосная упругая деформация приводит только к перераспределению носителей заряда между минимумами, которые смещаются в шкале энергий друг относительно друга, и не меняет формы изоэнергетических эллипсоидов в $n$-Ge. В результате проведенных исследований было получено не только качественное, но и количественное согласие экспериментальных данных с теоретическими расчетами, выполненными на основе формул теории анизотропного рассеяния, как видно из рис. 5-7.

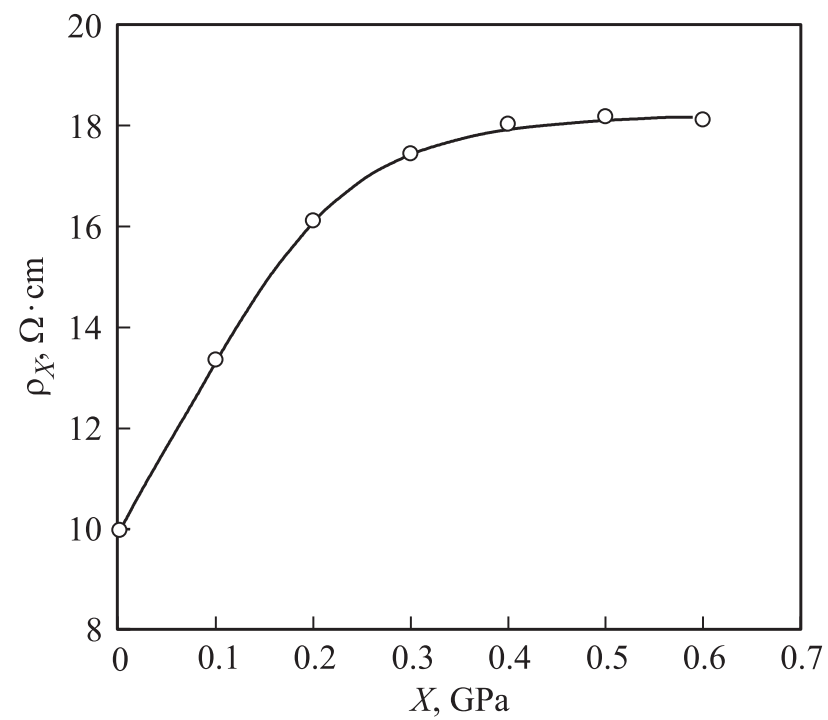

Рис. 5. Зависимость удельного сопротивления от деформирующего усилия, $\rho_{X}=f(X)$, в $n$-Ge $\left(n_{e}=1.48 \cdot 10^{13} \mathrm{~cm}^{-3}\right)$ при $77 \mathrm{~K}$ в условиях $\mathbf{X}\|\mathbf{J}\|[110]$ : точки - экспериментальные данные, сплошная кривая - результат теоретического расчета. 


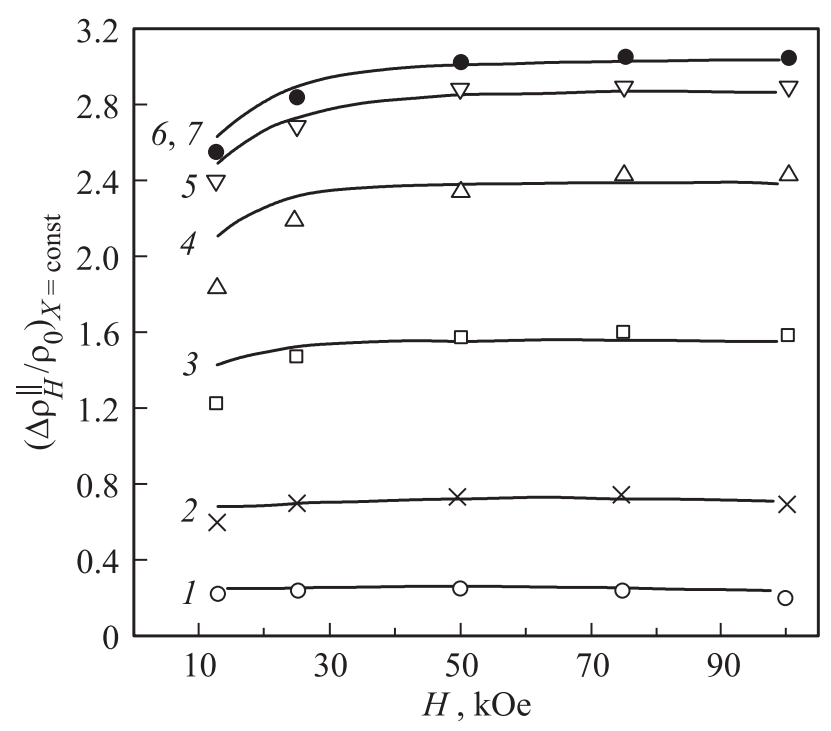

Рис. 6. Зависимости магнетотензосопротивления $\left(\Delta \rho_{H}^{\|} / \rho_{0}\right)_{X=\text { const }} \equiv\left(\rho_{X}^{H}-\rho_{X}^{0}\right) / \rho_{X}^{0}=f(H) \quad$ в $\quad n$-Ge $\quad\left(n_{e}=\right.$ $\left.=1.48 \cdot 10^{13} \mathrm{~cm}^{-3}\right)$ при $77 \mathrm{~K}$, измеренные в условиях $\mathbf{X}\|\mathbf{J}\| \mathbf{H} \|[110]$ при разных значениях $X$, ГПа: $1-0$, $2-0.1,3-0.2,4-0.3,5-0.4,6-0.5,7-0.6$. Точки экспериментальные данные, сплошные кривые - результаты теоретического расчета.

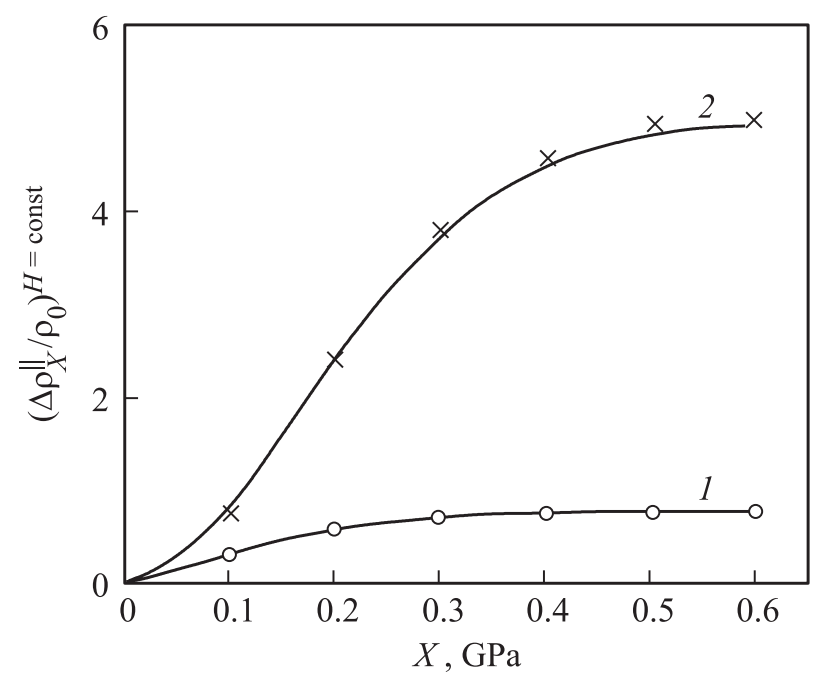

Рис. 7. Зависимости тензомагнетосопротивления $\left(\Delta \rho_{X}^{\|} / \rho_{0}\right)^{H=\text { const }} \equiv\left(\rho_{X}^{H}-\rho_{0}^{H}\right) / \rho_{0}^{H}=f(X) \quad$ в $\quad n$-Ge $\quad\left(n_{e}=\right.$ $\left.=1.48 \cdot 10^{13} \mathrm{~cm}^{-3}\right)$ при $77 \mathrm{~K}$, измеренные в условиях $\mathbf{X}\|\mathbf{J}\| \mathbf{H} \|[110]$ при разных напряженностях магнитного поля $H$, кЭ: $1-0,2-100$. Точки - экспериментальные данные, сплошные кривые - результаты теоретического расчета.

Показано, что в области насыщения магнетотензосопротивление в продольном магнитном поле при увеличении механического напряжения $\mathbf{X} \|[110]$ от 0 до 0.6 ГПа возрастает примерно в 10 раз (рис. 6, кривые $1-7$ ).

Проведено сопоставление экспериментальных данных, полученных в условиях $\mathbf{X}\|\mathbf{J}\| \mathbf{H} \|[111]$, с данными, полученными при $\mathbf{X}\|\mathbf{J}\| \mathbf{H} \|$ [110]. В случае направленной упругой деформации кристалла в кристаллографическом направлении [111] обеспечивалось деформационно-стимулированное переселение носителей из трех изоэнергетических эллипсоидов в один (в направлении большой оси которого прикладывалось к кристаллу внешнее сжимающее усилие $\mathbf{X}$ ). При $\mathbf{X} \|$ [110], в отличие от предыдущих условий, деформационно-стимулированное переселение электронов происходило из двух эллипсоидов, поднимающихся (в шкале энергий) вверх под влиянием деформации, в два опускающихся вниз эллипсоида. Именно поэтому в последнем случае деформационные изменения $\rho(X)$ как в отсутствие внешнего магнитного поля $H$ (рис. 5), так и при его наличии (рис. 6, 7) были по абсолютной величине значительно меньшими по сравнению с образцами, деформированными в кристаллографическом направлении [111] (рис. 1-3).

\section{3. Заключение}

Исследовано при $77 \mathrm{~K}$ тензосопротивление, тензомагнето- и магнетотензосопротивление $n$-Ge разной кристаллографической ориентации ([111] и [110]) при наличии классически сильного магнитного поля $(12.5 \leq H \leq 100$ кЭ) и без него $(H=0)$ и при направленной деформации сжатия в пределах $0 \leq X \leq 0.7$ ГПа. Обнаруженное количественное согласие теории с экспериментальными данными обосновывает предположение о том, что направленная упругая деформация только смещает одни минимумы энергии относительно других и обусловливает этим перераспределение носителей заряда между ними.

Установлено, что в условиях $\mathbf{X}\|\mathbf{J}\| \mathbf{H} \|[111]$ при значительных механических напряжениях $X$ сильно деформированный кристалл $n$-Ge не меняет значений $\rho$ даже под влиянием классически сильных магнитных полей (магнетотензосопротивление в сильно деформированном кристалле практически равно нулю).

Проведено сопоставление экспериментальных данных, полученных в условиях $\mathbf{X}\|\mathbf{J}\| \mathbf{H} \|[111]$, с данными, полученными при $\mathbf{X}\|\mathbf{J}\| \mathbf{H} \|[110]$. Установлено, что в последнем случае деформационные изменения $\rho(X)$ как в отсутствие внешнего магнитного поля $H$, так и при его наличии были по абсолютной величине значительно меньшими по сравнению с образцами, деформированными в кристаллографическом направлении [111].

\section{Приложение}

В Приложсении, согласно [9], представлены формулы теории анизотропного рассеяния, на основе которых проведены расчеты в предположении, что одноосная упругая деформация $n$-Ge влияет только на заполнение различных энергетических минимумов и не меняет 
общую концентрацию носителей заряда $n_{e}$, времена электрон-фононной релаксации и фононный спектр:

$$
\begin{gathered}
\rho_{X}^{H}=\frac{1}{\sigma_{11}^{(1)}}\left[\frac{K+2}{3}+\frac{N_{2}}{N_{1}} K \frac{I_{2}^{(2)}}{I_{1}^{(2)}}\right. \\
\left.+\left(\frac{e H \tau_{\perp}}{c m_{\perp}}\right)^{2}\left(\frac{I_{1}^{(1)}}{I_{2}^{(1)}}+\frac{N_{2}}{N_{1}} K \frac{I_{1}^{(2)}}{I_{2}^{(1)}}\right)\right]^{-1}, \\
\tau_{\|}^{(1)}=\frac{8 e^{2} N_{1} \tau_{\|} I_{2}^{(1)}}{3 \sqrt{\pi} m_{\|}} \\
a_{\|}=3.6 \cdot 10^{-17}, \quad a_{\perp}=3 \cdot 10^{-17}, \\
N_{1}=\frac{a_{\perp}}{1+\exp \left(E_{1}^{*}-E_{2}^{*}\right)}, \quad \frac{a_{2}}{N_{1}}=\exp \left(E_{1}^{*}-E_{2}^{*}\right) \\
\int_{n}^{(k)} \frac{x^{n} e^{-x} d x}{x+a_{k}}, \quad k=1,2, \\
E_{0}^{*}-E_{1}^{*}=-\frac{1}{3} \frac{\Xi_{u}}{k T} S_{44} X \\
a_{1}=\left(\frac{e H \tau_{\perp}}{c m_{\perp}}\right)^{2} \frac{2 K+1}{3 K}, \quad a_{2}=\left(\frac{e H \tau_{\perp}}{c m_{\perp}}\right)^{2} \frac{1}{m_{\perp}}
\end{gathered}
$$

Здесь $N_{0}$ - концентрация электронов в одном минимуме при отсутствии деформации (т.е. при $X=0$ ), $N_{1}$ и $\mathrm{N}_{2}$ - концентрации электронов соответственно в опускающихся и поднимающихся минимумах энергии в одноосно-деформированном кристалле, $E_{i}^{*}=E_{i} / k T-$ приведенный потенциал деформации $i$-го минимума, $\sigma_{i j}^{(n)}$ - компоненты тензора проводимости долины $n$ в лабораторной системе координат. В случае напряжения сжатия $(X<0)$ больцмановский переход носителей заряда (вследствие относительного смещения энергетических минимумов при деформации) приведет в результате к повышению их концентрации в тех эллипсоидах, в которых продольная ось образует малый угол с осью деформации. В предельном случае (при достаточно больших $X$ и относительно невысоких температурах $T$ ) электроны полностью перейдут из двух эллипсоидов, характеризующихся индексом 2, в минимумы с индексом 1. Поэтому насыщение функции $\rho_{X}=f(X)$ будет достигаться только при условии $2 N_{1}=4 N_{0}=n_{e} \equiv N$.

\section{Список литературы}

[1] Г.П. Гайдар, П.И. Баранский, В.В. Коломоец. Физика и химия твердого тела, 15 (1), 58 (2014).

[2] С.И. Будзуляк. Физика и химия твердого тела, $\mathbf{1 3}$ (1), 34 (2012).

[3] Ch.S. Smith. Phys. Rev., 94 (1), 42 (1954).

[4] C. Herring. Bell System Tech. J., 34 (2), 237 (1955).

[5] П.И. Баранский, А.В. Федосов, Г.П. Гайдар. Физические свойства кристаллов кремния и германия в полях эфбективного внешнего воздействия (Луцк, Надстырье, 2000).

[6] П.И. Баранский, А.В. Федосов, Г.П. Гайдар. Неоднородности полупроводников и актуальные задачи межсдефектного взаимодействия в радиационной физике и нанотехнологии (Киев-Луцк, РИО ЛГТУ, 2007).

[7] Г.П. Гайдар. Электронная обработка материалов, 51 (2), 85 (2015).

[8] П.И. Баранский, В.П. Клочков, И.В. Потыкевич. Полупроводниковая электроника. Справочник (Киев, Наук. думка, 1975).

[9] П.И. Баранский, И.С. Буда, И.В. Даховский, В.В. Коломоец. Электрические $u$ гальваномагнитные явления в анизотропных полупроводниках (Киев, Наук. думка, 1977).

[10] P.I. Baranskii, V.V. Kolomoets. Phys. Status Solidi B, 45 (1), K55 (1971).

Редактор Л.В. Шаронова

\section{Tensoresistance of $n$-Ge with different crystallographic orientation in the presence of a classically high magnetic field and without it}

\author{
G.P. Gaidar ${ }^{1}$, P.I. Baranskii ${ }^{2}$
}

${ }^{1}$ Institute for Nuclear Research, National Academy of Sciences of Ukraine, 03680 Kyiv, Ukraine

${ }^{2}$ V. Lashkaryov Institute of Semiconductor Physics, National Academy of Sciences of Ukraine, 03028 Kyiv, Ukraine

Abstract Changes of tensoresistance, tensomagnetoresistance and magnetotensoresistance were experimentally and theoretically studied in the wide ranges of the magnetic field intensities $0 \leq H \leq 100 \mathrm{kOe}$ and mechanical stresses $0 \leq X \leq 0.7 \mathrm{GPa}$ at $77 \mathrm{~K}$ under conditions of the nondegenerate statistics of the electron gas in $n$-Ge crystals with different crystallographic orientations. 